\title{
Voxel-Based Simulation Approach for Molecular Communications via Diffusion
}

\author{
H. Birkan Yilmaz ${ }^{\mathrm{a}, *}$, Xabier Gutierrez $^{\mathrm{b}}$, Ilker Demirkol $^{\mathrm{a}}$ \\ ${ }^{a}$ Dept. of Telematics Eng., Universitat Politecnica de Catalunya, Barcelona, Spain \\ ${ }^{b}$ Dept. of Telecommunications Eng., Universitat Politecnica de Catalunya, Barcelona, Spain
}

\begin{abstract}
Molecular communications via diffusion $(\mathrm{MCvD})$ systems are easily simulated for micro-scale topologies and applications. On the other hand, due to the high path loss, there is a need for the emission of a very large number of molecules to have a detectable signal for the macro-scale topologies. Therefore, the simulation of macro-scale $\mathrm{MCvD}$ systems or applications has its own challenges. In this work, a voxel-based simulator for $\mathrm{MCvD}$ is proposed and analyzed. The proposed simulator is able to consider a very large amount of molecules since it does not track every molecule, instead it simulates the aggregate behavior. We assess the correctness of such a simulation approach through comparative studies with a particle-based (i.e., per-molecule) simulation. We present the effect of voxel side-length on the modeling accuracy and devise a framework for selecting the optimal voxel side-length for high-accuracy simulations.
\end{abstract}

Keywords: Diffusion-based molecular communications, Voxel-based solver, Multi-grid neighbor method

\section{Introduction}

Collaborative behavior of very tiny and simple devices has the potential to be complex enough for advanced applications [1]. Therefore, the deriving applications of nanotechnology requires communication among these devices. It is not 5 effective to utilize the conventional communication techniques at small scales due to the inherent physical constraints such as the antenna size to wavelength ratio and some environments that are not suitable for propagation of electromagnetic waves $[2,3]$. In nature, molecular communications (MC) emerges as a solution to tackle with these hurdles at micro and macro scales as a result 10 of the evolutionary processes [4]. In MC, molecules are utilized to convey information among communication nodes at micro- and macro-scales. Some of

\footnotetext{
* Corresponding author

Email addresses: birkan.yilmaz@upc.edu (H. Birkan Yilmaz), xabier.gutierrez@alu-etsetb.upc.edu (Xabier Gutierrez), ilker.demirkol@entel.upc.edu (Ilker Demirkol)
}

Preprint submitted to Elsevier Nano Communication Networks

October 23, 2018 
the micro-scale MC systems can be listed as: quorum sensing in bacteria $[5,6]$, neurotransmitter signaling in neuromascular junctions [7], and inter-cell calcium signaling $[8,9]$. Examples of macro-scale MC systems in nature include 15 pheromone signaling among plants, chemical signaling among animals, and odor tracking of blue crabs in ocean. Utilizing MC is proposed for man-made and/or man-modified systems to counter the hurdles of the communication in small scales and the hurdles of some macro-scale environments where the conventional methods do not work effectively (e.g., networks of tunnels or salty water environments) $[1,2,3,10]$.

Molecular communications via diffusion $(\mathrm{MCvD})$ is one of the promising $\mathrm{MC}$ systems proposed especially due to its energy efficiency and its advantages in certain environments such as pipe networks filled with fluid $[1,3]$. In MCvD, information molecules propagate in the fluid environment via diffusion process

25 and they arrive at the receiver node in a probabilistic manner in which the received molecules constitute the received molecular signal $[2,3]$. Therefore, modeling the received molecular signal is one of the main challenges in $\mathrm{MCvD}$ with different environments and conditions.

In the literature, there are analytical channel models for simple and symmet-

so rical $\mathrm{MCvD}$ topologies with absorbing and passive receivers: absorbing receiver in 1-dimensional (1D) environment [11]; absorbing spherical receiver in 3D environment [12]; absorbing receptors on a spherical receiver [13]; passive spherical transmitter and receiver in $3 \mathrm{D}$ environment $[14,15,16]$. In addition, simulators are highly utilized for complex topologies and for verifying analytical models 35 of simple topologies in micro-scales $[17,18,19,20]$. Simulators that are introduced for $\mathrm{MCvD}$ are designed for micro-scale environments effectively -with tracking feasible number of molecules. On the contrary, simulators for macroscale MCvD systems have not been developed due to the hurdles caused by high path loss. For macro-scale applications, significant amount of molecules should 40 be released and tracked, therefore a new approach should be considered for the $\mathrm{MCvD}$ simulations at macro-scale.

Computer simulations in fluid dynamics are studied for reaction-diffusion processes in biochemical applications [21, 22, 23, 24]. The Gillespie method was proposed in 1976 [25] and became popular in 1977 with its application to chem-

${ }_{45}$ ical and biochemical systems of reactions based on the diffusion reaction master equations [26]. Then, the Gillespie method was extended to multi-particle systems and adapted to the lattice gas automata model by Chopard et al. [21]. The Gillespie multi-particle method utilizes discretization in time and space, and each lattice site holds a discrete number of uniformly distributed particles. It 50 employs the diffusion process for particles to distribute them randomly among the nearest lattice site neighbors at certain time instances. In this method, however, the main concern is accurately mimicking the reactions at local sites. The diffusion step is followed by the reaction step at each local grid structure. Chopard et al. eliminated the checkerboard effect, at the diffusion step, by in55 troducing still molecules [21]. However, the probability of staying in a lattice is left as a system parameter. In this work we define the probability of staying in a lattice with a more structured framework. Moreover, the time resolution in 
the Gillespie multi-particle method is defined as $\lambda^{2} / 6 D$, where $\lambda$ is the lattice side length and $D$ is the diffusion coefficient. However, the defined time resolution can be considered for the systems in equilibrium. For communication purposes the accuracy of the time dependent received signal should be analyzed with different simulation time step and lattice side length values. The Gillespie multi-particle gas automata method is utilized in MC domain [27]. However, authors considered the default time resolution and $\lambda$ values, which resulted in

65 theory and simulation mismatch. In [20], a hybrid simulation approach (in terms of utilizing mesoscopic and microscopic approaches) is proposed and named as AcCoRD. AcCoRD utilizes the tau-leaping concept and the reactions in the environment without any consideration on the optimal lattice size.

In this work, we propose to use a grid-based (voxel-based, where a voxel

70 stands for a partition structure of the environment that can be a cube or a square depending on the dimension of the environment) MCvD simulator that can simulate a very large number of molecules, since it does not track the molecules individually. After deriving the transition probabilities between the voxels analytically, the aggregate behavior of the molecules is simulated at each iteration

75 for the transition from one voxel to another. The accuracy of the received molecular signal is evaluated via comparison with particle-based simulations for different scenarios. Moreover, the framework for selecting the grid size for the accurate simulations is presented by also considering the probability of staying in a voxel. Note that the voxel-based simulator is independent from the num-

so ber of released molecules and evaluates the mean received signal at the receiver from a communications perspective. This work is an extension of a preliminary work in [28] with an improved mathematical framework considering also $3 \mathrm{D}$ environments, and introducing a new framework for selecting the optimum voxel side-length. In our previous study, we presented only the effect of voxel side-length on the model accuracy.

\section{System Model: Molecular Communications via Diffusion}

MCvD system mainly consists of a transmitter node, a fluid environment, the information molecules, and a receiver node $[1,2,3]$. The information is transferred via the emitted molecules that have been modulated by a predeter-

90 mined modulation scheme (e.g., concentration shift keying (CSK) and molecular shift keying (MoSK)) [29]. We focus on the received molecular signal which is mainly affected by the system topology and the diffusion model.

\subsection{Topology Model}

We consider an $\mathrm{MCvD}$ system with a point transmitter and an absorbing 95 receiver with any given shape in a 2D/3D environment, as illustrated in Fig. 1 for 3D environment. The point source and the receiver both reside in a fluid propagation medium. It is assumed that the medium is unconfined, thus extending to infinity in all dimensions and the interactions between the diffusing molecules are ignored. Emitted molecules propagate by the diffusion process 


\section{Emission}

Propagation

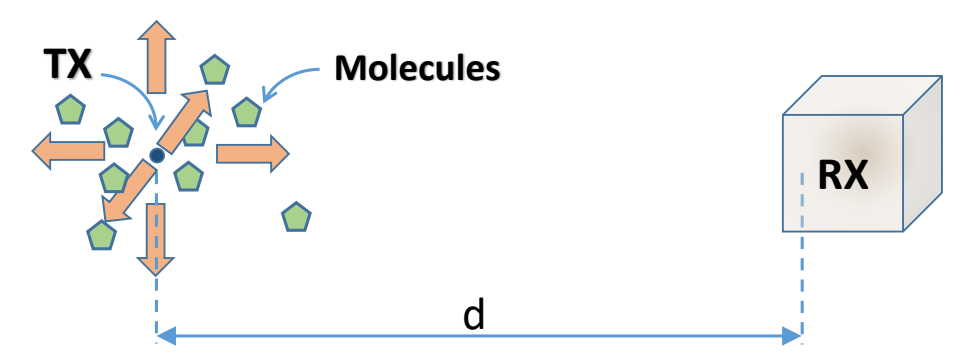

Figure 1: Processes, entities, and system model for MCvD with a point transmitter (TX) at time $t$ and with the location vector $r$ starting from the emission point.

We assume that the receiver is a perfectly absorbing node and whenever a molecule hits the surface of the receiver node, it is removed from the environment. Therefore, each molecule can contribute to the received signal only once for an absorbing receiver. When these dynamics are introduced as initial and boundary conditions, the solution to this differential equation system is enabled for symmetrical and simple topologies and is solved for the cumulative mean fraction of molecules that hit the receiver in $n$-dimensional space until time $t$, which is denoted by $\tilde{\mathbf{F}}_{\text {hit }}^{\text {nD }}(t)$. After obtaining $\tilde{\mathbf{F}}_{\text {hit }}^{\text {nD }}(t)$, the mean number of received molecules between $t_{1}$ and $t_{2}$ (i.e., $\tilde{\mathbf{N}}_{\mathrm{Rx}}^{\mathrm{nD}}\left(t_{1}, t_{2}\right)$ ) can be evaluated by:

$$
\tilde{\mathbf{N}}_{\mathrm{Rx}}^{\mathrm{nD}}\left(t_{1}, t_{2}\right)=\mathbf{N}_{\mathrm{Tx}} \times\left(\tilde{\mathbf{F}}_{\text {hit }}^{\mathrm{nD}}\left(t_{2}\right)-\tilde{\mathbf{F}}_{\text {hit }}^{\mathrm{nD}}\left(t_{1}\right)\right)
$$

where $\mathbf{N}_{\text {Tx }}$ is the number of emitted molecules. $\tilde{\mathbf{F}}_{\text {hit }}^{3 \mathrm{D}}(t)$ is presented and analyzed for a perfectly absorbing spherical receiver in [12]. The authors present the formula for the mean fraction of molecules that hit the receiver until time $t$, as 
follows:

$$
\tilde{\mathbf{F}}_{\text {hit }}^{3 \mathrm{D}}(t)=\frac{r^{\mathrm{Rx}}}{d+r^{\mathrm{Rx}}} \operatorname{erfc}\left(\frac{d}{\sqrt{4 D t}}\right)
$$

where $d$ represents the distance between the transmitter and the receiver surface, $r^{\mathrm{Rx}}$ is the receiver radius, and $\operatorname{erfc}($.$) represents the complementary error$ function. For the point transmitter and spherical absorbing receiver case, the solution for the system of differential equations is enabled due to the circular symmetry. In addition, it is feasible to implement particle-tracking based simulators to verify the derived analytical models. On the other hand, for more complex topologies, it is harder to derive the number of received molecules analytically. Moreover, for some cases such as irregular shapes or long distances, also the simulations become infeasible due to increased complexity and requirement of very high number of molecules.

\section{Simulation Approaches}

Main simulation approaches are presented in Fig. 2 as: particle-tracking and voxel-tracking. In particle-tracking case, all the molecules are tracked in time (i.e., coordinates of all the molecules are tracked at each time instance). On the other hand, voxel-tracking based simulator tracks the voxels in discretized time (i.e., the mean aggregate number of molecules in each voxel is tracked at each time instance).

For macro-scale MCvD applications, $\tilde{\mathbf{F}}_{\text {hit }}^{3 \mathrm{D}}(t)$ becomes extremely small, which requires huge number of molecules to be tracked for statistically meaningful results. Such a high number of molecules becomes intractable for macro-scale $\mathrm{MCvD}$ applications, which in turn creates the scalability problem for particletracking based simulators. To tackle this challenge, we propose an effective way to simulate macro-scale MCvD systems.

\subsection{Particle-tracking Based Simulation}

The dynamics of diffusion can be described by Brownian motion. Simulating the Brownian motion includes consecutive steps in an $n$-dimensional space that obeys Wiener process dynamics. For a particle-tracking based simulation, time is discretized with a sufficiently small time intervals $(\Delta t)$ and at each time interval molecules take random steps in all dimensions that follows:

$$
\Delta r_{i} \sim \mathscr{N}(0,2 D \Delta t)
$$

where $r_{i}$ is the $i^{\text {th }}$ dimension, $D$ is the diffusion coefficient, and $\mathscr{N}\left(\mu, \sigma^{2}\right)$ is the Gaussian distribution with mean $\mu$ and the variance $\sigma^{2}$. After each random step, molecule positions are checked for possible hit to receiver and if molecules are inside the receiver they are recorded for that time instance. Hence, we obtain an empirical estimate for the received molecular signal that includes diffusion noise (i.e., $\hat{\mathbf{N}}_{\mathrm{Rx}}^{\mathrm{nD}}\left(t_{1}, t_{2}\right)$ for $n$-dimensional space). 


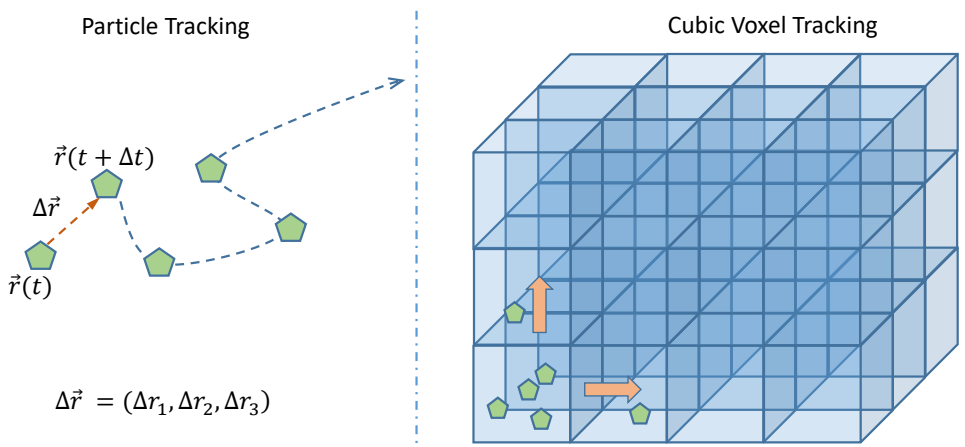

Figure 2: Two main simulation approaches for MCvD systems. In particle-tracking based simulations, a random displacement according to diffusion dynamics is generated and added to the current position of the diffusion molecules at each simulation time step. In voxel-tracking based simulations, environment is divided into voxels and the transitions between voxels are simulated according to diffusion dynamics.

For micro-scale environments, it is feasible to track all the emitted molecules, which is in the order of millions. However, for macro-scale $\mathrm{MCvD}$ applications, we have to consider very large amount of molecules (e.g., more than Avogadro

\subsection{Voxel-tracking Based Simulation}

In voxel-tracking based simulator, the environment is divided into voxels (i.e., square or cubes in $2 \mathrm{D}$ or $3 \mathrm{D}$ environments, respectively) and molecules are released from a point in the environment. For each voxel, only the total number of molecules reside in that voxel is tracked for each time instance in an aggregate manner [28]. Moreover, we assume that the molecules in a voxel are distributed homogeneously. The transition between the voxels are simulated as follows for the 2D environment: For the molecules of a given voxel, the molecules either stay at the same voxel with a probability of stay or move to the 4-neighbors (the vertical and horizontal neighbors) with equal transition probabilities. Alternatively, 8-neighbors are considered (by including the neighbors at the diagonals) with a different transition probability for the diagonal neighbors. In the $3 \mathrm{D}$ environment, the molecules might move to the 6 -neighbors according to a common transition probability or 26-neighbors with three types of transition probabilities [28].

For the aggregate molecule transition/movement concept in $2 \mathrm{D}$ or $3 \mathrm{D}$ environment, first, we evaluate the probability of staying $\left(\mathbf{P}_{\text {stay }}^{2 \mathrm{D}}\right.$ or $\mathbf{P}_{\text {stay }}^{3 \mathrm{D}}$, respec- 
tively) for the molecules in a voxel -by utilizing (4)- as follows:

$$
\begin{aligned}
& \mathbf{P}_{\text {stay }}^{2 \mathrm{D}}=\frac{1}{\lambda^{2}} \int_{0}^{\lambda} \int_{0}^{\lambda} \mathbf{P}_{\Delta r_{1}} \mathbf{P}_{\Delta r_{2}} d r_{1} d r_{2} \\
& \mathbf{P}_{\text {stay }}^{3 \mathrm{D}}=\frac{1}{\lambda^{3}} \int_{0}^{\lambda} \int_{0}^{\lambda} \int_{0}^{\lambda} \mathbf{P}_{\Delta r_{1}} \mathbf{P}_{\Delta r_{2}} \mathbf{P}_{\Delta r_{3}} d r_{1} d r_{2} d r_{3} \\
& \mathbf{P}_{\Delta r_{i}}=\mathbf{P}\left(-r_{i}<\Delta r_{i}<\lambda-r_{i}\right) \\
& \mathbf{P}_{\Delta r_{i}}=\frac{1}{2}\left[\operatorname{erf}\left(\frac{\lambda-r_{i}}{\sqrt{4 D \Delta t}}\right)-\operatorname{erf}\left(\frac{-r_{i}}{\sqrt{4 D \Delta t}}\right)\right]
\end{aligned}
$$

where $\lambda$ and $\Delta t$ are the length of the voxel sides and the simulation time step size, respectively. After the integration, $\mathbf{P}_{\text {stay }}^{2 \mathrm{D}}$ can be written as follows:

$$
\begin{aligned}
\mathbf{P}_{\text {stay }}^{2 \mathrm{D}} & =\frac{e^{-\frac{2 \lambda^{2}}{S^{2}}}\left[S e^{\lambda^{2} / S^{2}}-\lambda \sqrt{\pi} \operatorname{erf}\left(\frac{\lambda}{S}\right) e^{\lambda^{2} / S^{2}}-S\right]^{2}}{\lambda^{2} \pi} \\
S & =\sqrt{4 D \Delta t}
\end{aligned}
$$

After evaluating $\mathbf{P}_{\text {stay }}^{2 \mathrm{D}}$ or $\mathbf{P}_{\text {stay }}^{3 \mathrm{D}}$, we derive the aggregate transition probabilities that is depending on the number of neighbors that we use in the passage.

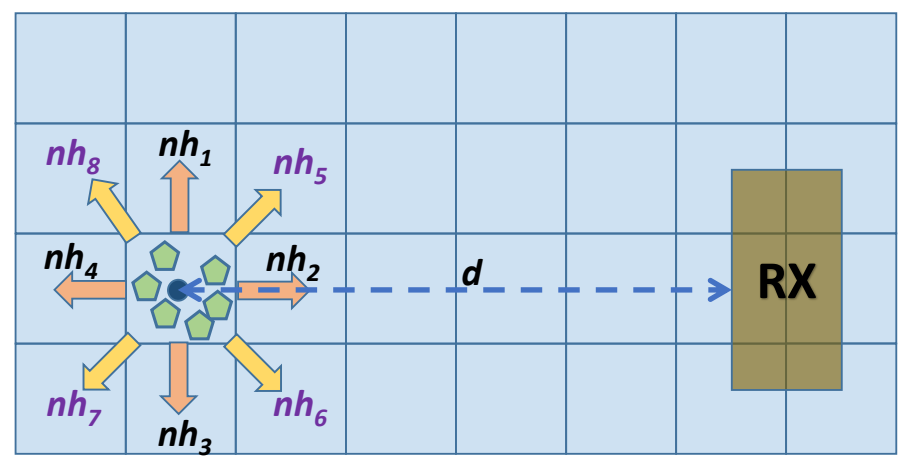

Figure 3: Example topology for voxel neighbors in 2D MCvD system. Neighbors are shown for the voxel that has the transmitter point. Neighbors that are denoted by $n h_{1}$ to $n h_{4}$ are the direct neighbors and $n h_{5}$ to $n h_{8}$ are the diagonal neighbors for this example topology. Evaluations and modeling are invariant of the neighbor indexing system, for which we used a clockwise order.

In Fig. 3, an MCvD system is presented for an example topology for a $2 \mathrm{D}$ environment. The neighboring voxels of a selected center voxel are also presented for defining the neighbors. The system model with 4-neighbors in $2 \mathrm{D}$ environment is also called the neighborhood model without diagonal neighbors. In $2 \mathrm{D}$ environment, if we use 4-neighbors then $1-\mathbf{P}_{\text {stay }}^{2 \mathrm{D}}$ is distributed equally to all 4-neighbors as follows:

$$
\mathbf{P}_{\text {trans }}^{2 \mathrm{D}}\left(n h_{i}\right)= \begin{cases}\frac{1-\mathbf{P}_{\text {stay }}^{2 \mathrm{D}}}{4} & \text { for } i=1,2,3,4 \\ 0 & \text { for } i=5,6,7,8\end{cases}
$$


where $\mathbf{P}_{\text {trans }}^{2 \mathrm{D}}\left(n h_{i}\right)$ represents the molecule's probability of passing to $i^{\text {th }}$ neighbor. The system model with 8-neighbors in 2D environment is called the neighborhood model with diagonal neighbors. In $2 \mathrm{D}$ environment, if we use 8-neighbors then $1-\mathbf{P}_{\text {stay }}^{2 \mathrm{D}}$ is distributed to direct neighbors and diagonal neighbors as follows:

$$
\begin{aligned}
\mathbf{P}_{\text {trans }}^{2 \mathrm{D}}\left(n h_{i}\right) & = \begin{cases}\alpha_{1}\left(1-\mathbf{P}_{\mathrm{stay}}^{2 \mathrm{D}}\right) & \text { for } i=1,2,3,4 \\
\alpha_{2}\left(1-\mathbf{P}_{\mathrm{stay}}^{2 \mathrm{D}}\right) & \text { for } i=5,6,7,8\end{cases} \\
\alpha_{1} & =\frac{1}{4} \frac{\Phi_{\text {direct }}^{2 \mathrm{D}}}{\Phi_{\text {direct }}^{2 \mathrm{D}}+\Phi_{\mathrm{diag}}^{2 \mathrm{D}}} \\
\alpha_{2} & =\frac{1}{4} \frac{\Phi_{\text {diag }}^{2 \mathrm{D}}}{\Phi_{\text {direct }}^{2 \mathrm{D}}+\Phi_{\text {diag }}^{2 \mathrm{D}}}
\end{aligned}
$$

where

$$
\begin{aligned}
\Phi_{\text {direct }}^{2 \mathrm{D}} & =\frac{1}{\lambda^{2}} \int_{0}^{\lambda} \int_{0}^{\lambda} \mathbf{P}_{\Delta r_{1}}(0, \lambda) \mathbf{P}_{\Delta r_{2}}(\lambda, 2 \lambda) d r_{1} d r_{2} \\
\Phi_{\text {diag }}^{2 \mathrm{D}} & =\frac{1}{\lambda^{2}} \int_{0}^{\lambda} \int_{0}^{\lambda} \mathbf{P}_{\Delta r_{1}}(\lambda, 2 \lambda) \mathbf{P}_{\Delta r_{2}}(\lambda, 2 \lambda) d r_{1} d r_{2} \\
\mathbf{P}_{\Delta r_{i}}\left(r_{\text {left }}, r_{\text {right }}\right) & =\frac{1}{2}\left[\operatorname{erf}\left(\frac{r_{\text {right }}-r_{i}}{\sqrt{4 D \Delta t}}\right)-\operatorname{erf}\left(\frac{r_{\text {left }}-r_{i}}{\sqrt{4 D \Delta t}}\right)\right]
\end{aligned}
$$

Similarly, we can derive the transition probabilities to the neighbors for $3 \mathrm{D}$ environment. At each time step $(\Delta t)$, the number of molecules that leave the voxels is determined and they are distributed to the neighbor voxels. For the arrival process, we assume perfect absorption. Whenever molecules reach to the receiver voxels, they are removed from the environment. Note that if the receiver body is partially intersecting with a voxel in the environment (as in Fig. 3), then the molecules are received proportional to volumes/areas in $3 \mathrm{D} / 2 \mathrm{D}$ environments.

Since the voxel-tracking based simulator keeps track of the voxels (not the molecules individually), the time complexity is in the order of the number of voxels in the environment. However, in voxel-tracking based simulator, there is a hyper-parameter that needs to be optimized: length of the voxel sides

${ }_{170}(\lambda)$. If the voxel side-length is too small then the simulator will not be able to capture the diffusion behavior of molecules that would be moving to two voxel distant places. On the other hand, a large voxel-side length results in a higher aggregation, which will again deteriorate the simulator accuracy. In the following, we investigate this trade-off through numerical evaluations.

\section{Simulation Results}

\subsection{Parameters and Performance Metrics}

In this work, we compare particle-tracking and voxel-tracking simulators by considering a fully absorbing receiver in $2 \mathrm{D}$ and $3 \mathrm{D}$ environments. Simulation 
parameters are listed in Table 1 . At the receiver side, the number of received molecules $\left(\mathbf{N}_{\mathrm{Rx}}\right)$ is recorded at each time step, which corresponds to the received signal. We compare the received molecular signals generated by both simulators. We applied Kolmogorov-Simirnov (KS) test on the received molecular signals (i.e., the number of received molecules versus time) with significance level 0.05 for comparing particle-tracking and voxel-tracking simulators, while considering the particle-tracking based simulations as the ground truth. We also evaluated normalized mean squared error (NMSE) for finding the optimal $\lambda$, which is denoted as $\lambda_{\mathrm{OPT}}$. Then, we analyzed the effect of system parameters on $\lambda_{\mathrm{OPT}}$.

Table 1: Simulation parameters

\begin{tabular}{ll}
\hline Parameter & Value \\
\hline Distance $(d)$ & $\{7,9,18\} \mu \mathrm{m}$ \\
Diffusion coefficient $(D)$ & $100 \sim 400 \mu \mathrm{m}^{2} / \mathrm{s}$ \\
Simulation time step $(\Delta t)$ & $0.00005 \sim 0.001 \mathrm{~s}$ \\
Number of released molecules $\left(N^{\mathrm{Tx}}\right)$ & 100000 \\
Number of Replications & 100 \\
\hline
\end{tabular}
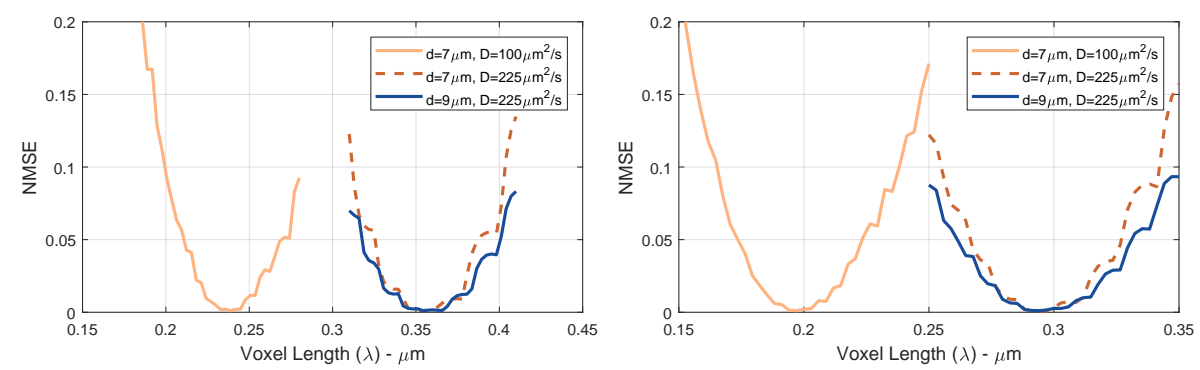

(a) NMSE plot for 2D environment (without (b) NMSE plot for 2D environment (with didiagonal neighbors).

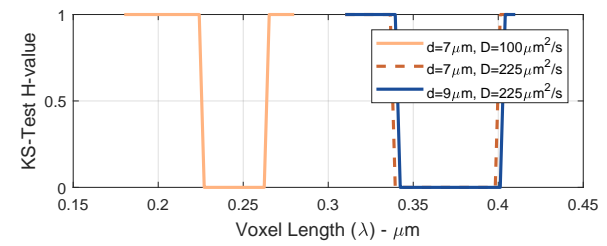
agonal neighbors).

(c) KS-test plot for 2D environment (without (d) KS-test plot for 2D environment (with didiagonal neighbors). agonal neighbors).

Figure 4: NMSE and KS-test plots for 2D environment with different neighborhood models. Received signals from particle-based and voxel-based simulations are compared. Best voxel side-lengths in terms of NMSE lies in fail-to-reject region of the KS-test for both systems. 


\subsection{Analysis on Neighborhood Model}

190 neighborhood model. In Fig. 4, the NMSE and KS-test results are presented for both systems (i.e., the systems with and without diagonal neighbors for the voxel-based simulations). If we focus on $\lambda_{\mathrm{OPT}}$ in Fig. $4 \mathrm{a}$ and Fig. 4b separately, the optimal voxel side-length shifts right when $D$ increases and is not affected by diagonal neighbors since a larger area is covered at any given step. To reduce the error due to the redistributed molecules from the diagonals, the system without diagonal neighbors requires two simulation steps to see the effect in the diagonal voxels. For both of the systems, $\lambda_{\mathrm{OPT}}$ in terms of NMSE lies in the fail-to-reject NMSE difference between the two voxel-based systems are negligible, hence in the remaining of this paper we only consider the neighborhood system without the diagonal neighbors due to its simplicity.

\subsection{Analysis on Received Signal}

We analyze the received signal based on the output of particle-based simulator and the voxel-based simulator without diagonal neighbors, by varying the scenario parameters such as distance and diffusion coefficient (see Fig. 5 and Fig. 6). In Fig. 5, we first observe that the voxel-based simulations with $\lambda_{\mathrm{OPT}}$ in $2 \mathrm{D}$ environments gives the best fit to the received signal of particlewhen we have $\lambda$ away from the $\lambda_{\mathrm{OPT}}$. The conventional $\lambda$ value for cellular automata-based simulators is chosen as $\sqrt{4 D \Delta t}$ for $2 \mathrm{D}$ environment in the Gillespie multi-particle method [21, 22, 25, 27]. We denote this choice of $\lambda$ as $\lambda_{\mathrm{GMP}}$ in performance plots. As was mentioned before, $\lambda_{\mathrm{GMP}}$ is effective for 215 steady state regimes $[21,22,25,27]$. However, the time dependent received signal accuracy in non-steady state regimes is also important for MCvD systems.

Similarly, in Fig. 6, the received signals in 3D environments are presented for different system parameters. We again observe that the voxel-based simulations 20 with $\lambda_{\mathrm{OPT}}$ gives the best fit to the received signal of particle-based simulations. Note that the $\lambda_{\mathrm{OPT}}$ values for $3 \mathrm{D}$ environment are different than those of the $2 \mathrm{D}$ environments. When we compare Fig. 5 and Fig. 6 , we also observe that the number of received molecules decreases for $3 \mathrm{D}$ environments due to one extra spreading dimension. For 3D environments, the conventional $\lambda$ value is $\sqrt{6 D \Delta t}$ in cellular automata-based simulators that are inspired by the Gillespie multiparticle method $[21,22,25,27]$. Similarly to the 2D environments case, the received signal in $3 \mathrm{D}$ environments that is corresponding to $\lambda_{\mathrm{GMP}}$ does not fit well to the ground truth especially in the peak region, which is a crucial data for the MCvD systems. Therefore, we focus on using $\lambda_{\mathrm{OPT}}$ and finding a closed 

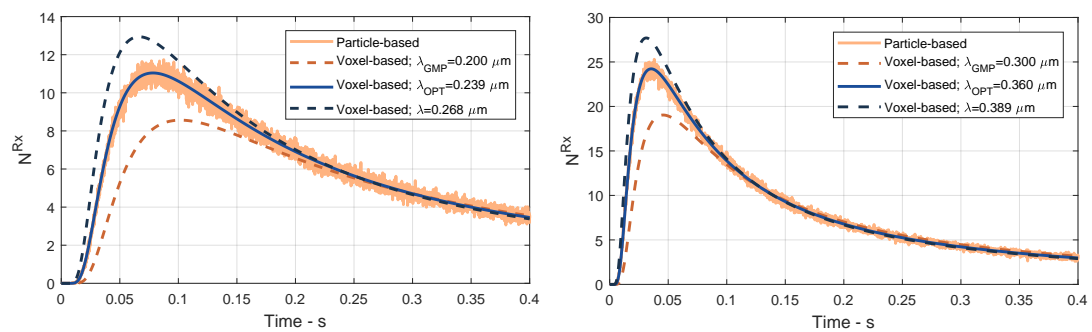

(a) Received signal in $2 \mathrm{D}$ environment (b) Received signal in $2 \mathrm{D}$ environment $\left(d=7 \mu \mathrm{m}, D=100 \mu \mathrm{m}^{2} / \mathrm{s}\right)$.

$\left(d=7 \mu \mathrm{m}, D=225 \mu \mathrm{m}^{2} / \mathrm{s}\right)$.

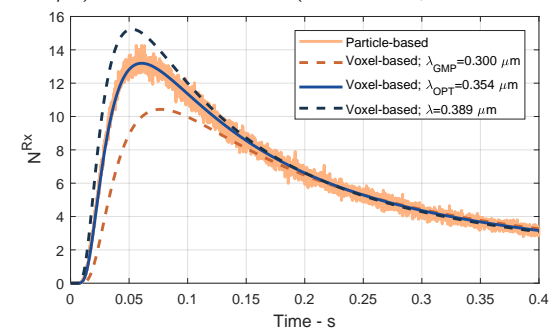

(c) Received signal in $2 \mathrm{D}$ environment $\left(d=9 \mu \mathrm{m}, D=225 \mu \mathrm{m}^{2} / \mathrm{s}\right)$.

Figure 5: Received signal plots for different parameters in 2D environments. Received signals corresponding to different voxel side-lengths are compared with $\lambda_{\mathrm{OPT}}(\Delta t=0.0001 \mathrm{~s})$.
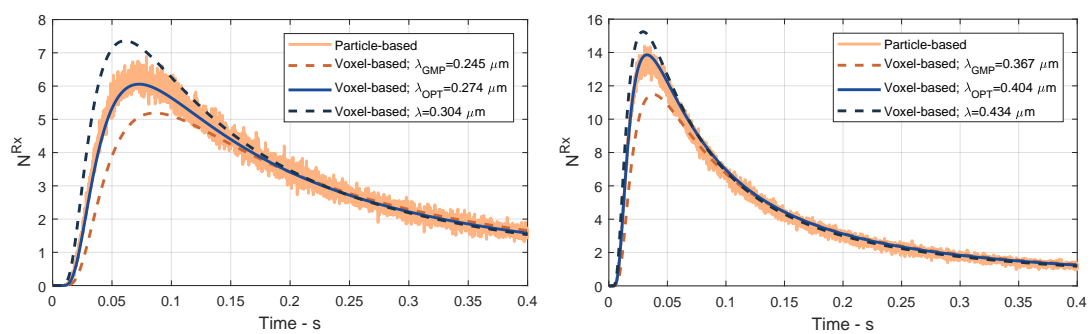

(a) Received signal in 3D environment (b) Received signal in 3D environment $\left(d=7 \mu \mathrm{m}, D=100 \mu \mathrm{m}^{2} / \mathrm{s}\right)$. $\left(d=7 \mu \mathrm{m}, D=225 \mu \mathrm{m}^{2} / \mathrm{s}\right)$.

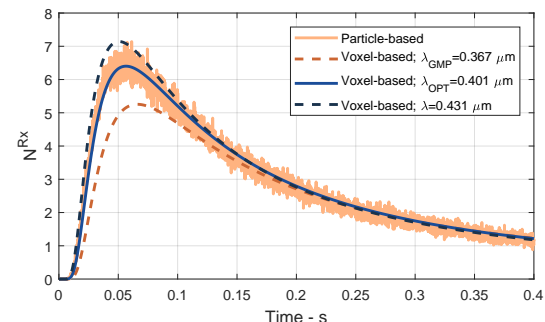

(c) Received signal in $3 \mathrm{D}$ environment (d $=9 \mu \mathrm{m}, D=225 \mu \mathrm{m}^{2} / \mathrm{s}$ ).

Figure 6: Received signal plots for different parameters in $3 \mathrm{D}$ environments. Received signals corresponding to different voxel lengths are compared with $\lambda_{\mathrm{OPT}}(\Delta t=0.0001 \mathrm{~s})$. 


\subsection{Analysis on Optimal Voxel Side-length}

In this section, we analyze the effect of $D$ and $\Delta t$ on the optimal voxel side-length since the step size of the molecules are affected by $D$ and $\Delta t$. We first focus on the effect of $D$ which is followed by the effect of $\Delta t$. Later on we propose a method to represent the framework for finding $\lambda_{\mathrm{OPT}}$ in a more generic way. In Fig. 7, the diffusion coefficient versus the optimum voxel side-length

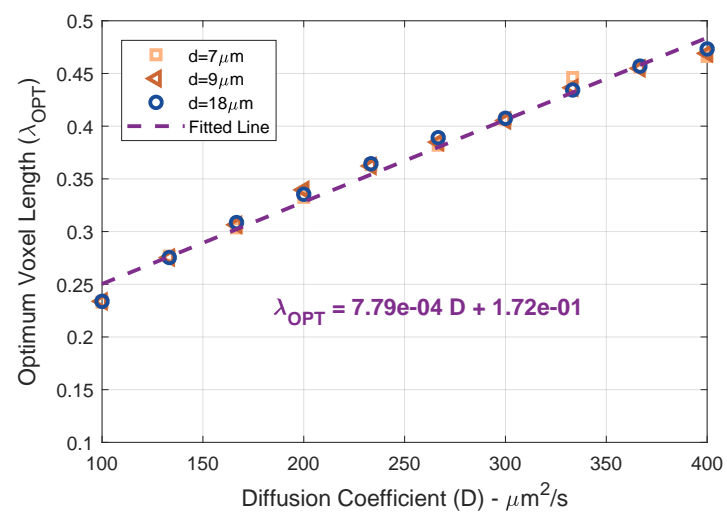

Figure 7: Optimal voxel side-length plot for $2 \mathrm{D}$ environment and the system without diagonal neighbors. A linear model is fitted to obtain $\lambda_{\mathrm{OPT}}$ in terms of $D(\Delta t=0.0001 \mathrm{~s})$.

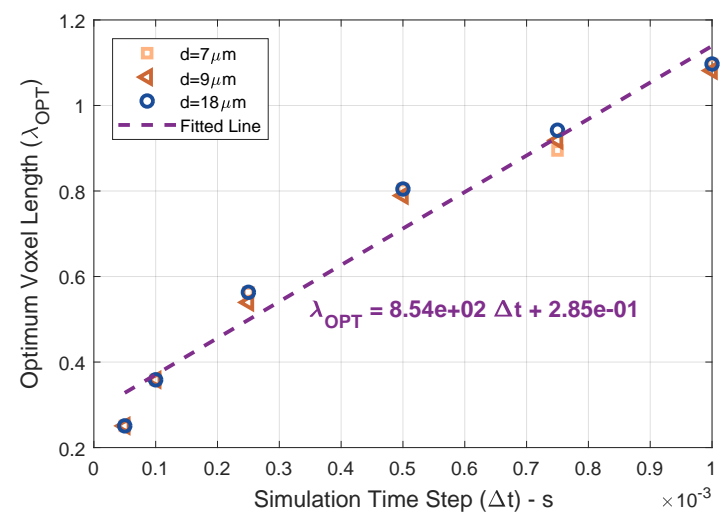

Figure 8: Optimal voxel side-length plot for $2 \mathrm{D}$ environment and the system without diagonal neighbors. A linear model is fitted to obtain $\lambda_{\mathrm{OPT}}$ in terms of $\Delta t\left(D=225 \mu \mathrm{m}^{2} / \mathrm{s}\right)$.

is depicted for $\Delta t=0.0001 \mathrm{~s}$. We observe that the optimum voxel side-length does not change when the distance is varied. On the other hand, $D$ affects the optimum voxel side-length linearly. Therefore, a linear model is fitted to the experimental data and shown in Fig. 7. Similarly, when we consider the effect of $\Delta t$ on the optimal voxel side-length, in Fig. 8, we observe that the optimum voxel side-length does not change with varying distance. On the other hand, 
$\Delta t$ affects the optimum voxel side-length linearly. Therefore, a linear model is fitted to the experimental data and shown in Fig. 8.

Let $\mathbf{P}_{\text {stay, OPT }}^{2 \mathrm{D}}$ denote $\mathbf{P}_{\text {stay }}^{2 \mathrm{D}}$ for a given $\lambda_{\mathrm{OPT}}$ in $2 \mathrm{D}$ environment. We discovered that $\mathbf{P}_{\text {stay,OPT }}^{2 \mathrm{D}}$ does not vary between scenarios with different system parameters, i.e. for different $\lambda_{\mathrm{OPT}}$. Therefore, for the optimal voxel side-lengths in $2 \mathrm{D}$ environment, $\mathbf{P}_{\text {stay,Opt }}^{2 \mathrm{D}}$ is not affected by $D$ or $\Delta t$. This is shown in Fig. 9, where $\mathbf{P}_{\text {stay, OPT }}^{2 \mathrm{D}}$ values are presented for different distances and diffusion coefficients. It can be clearly seen that the $\mathbf{P}_{\text {stay,OPT }}^{2 \mathrm{D}}$ is invariant for varying distance and diffusion coefficient values. Such invariant $\mathbf{P}_{\text {stay, OPT }}^{2 \mathrm{D}}$ value allows us to calculate $\lambda_{\mathrm{OPT}}$ by using the numerical evaluation of the inverse function for the formulation of $\mathbf{P}_{\text {stay }}^{2 \mathrm{D}}$ in (5). Fixing the system dynamics (e.g., using voxel-based simulator that does not consider the diagonal neighbors) leads to a single $\mathbf{P}_{\text {stay, OPT }}^{2 \mathrm{D}}$ hence an effective way to find optimum voxel side-length.

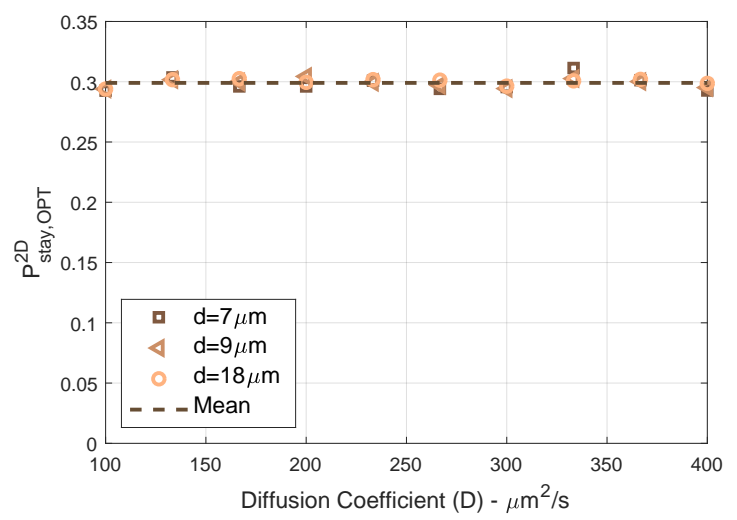

Figure 9: Probability of stay plot for 2D environments (model without diagonal neighbors)

\section{Conclusion}

In this work, we introduced the voxel-based simulator for MCvD systems especially for macro-scale applications. In macro-scale MCvD applications, there is a need for simulating a very large number of molecule movements due to high path loss. Therefore, we proposed a specialized voxel-based simulator for MCvD systems that is independent of the number of emitted molecules and that is keeping track of aggregate movements via voxel transition probabilities. Therefore, the voxel-tracking based simulator is capable of simulating huge amount of molecules since the voxel-tracking based simulations are simulating the envi265 ronment and independent of the number of emitted molecules. The proposed voxel-tracking based simulator is validated via particle-tracking based simulations. We proposed and assessed two voxel-based simulation frameworks that define different neighborhood models and showed that the difference is negligible in terms of accuracy and we continued with the one that does not use 
diagonal voxels due to its simplicity. In the literature, the voxel side-length is commonly chosen as in the Gillespie method and it is sufficient to model the received signal in steady state, however we showed the existence of the optimal voxel side-length that is capable of modeling the peak region of the received signal. Finally, we derived a mathematical framework for selecting the optimal voxel side-length, which minimizes the NMSE error of the simulator.

\section{ACKNOWLEDGEMENT}

This research is supported in part by the Government of Catalonia's Secretariat for Universities and Research via the Beatriu de Pinós postdoctoral programme and Spanish Government, MINECO, through project RYC-201313029 .

\section{References}

\section{References}

[1] I. F. Akyildiz, F. Brunetti, C. Blazquez, Nanonetworks: A new communication paradigm, Elsevier Comput. Netw. 52 (12) (2008) 2260-2279.

[2] T. Nakano, A. W. Eckford, T. Haraguchi, Molecular communication, Cambridge University Press, 2013.

[3] N. Farsad, H. B. Yilmaz, A. Eckford, C.-B. Chae, W. Guo, A comprehensive survey of recent advancements in molecular communication, IEEE Commun. Surveys Tuts. 18 (3) (2016) 1887-1919.

[4] M. D. Hauser, The evolution of communication, MIT press, 1996.

[5] L. C. Cobo, I. F. Akyildiz, Bacteria-based communication in nanonetworks, Elsevier Nano Commun. Netw. 1 (4) (2010) 244-256.

[6] S. Abadal, I. F. Akyildiz, Automata modeling of quorum sensing for nanocommunication networks, Elsevier Nano Commun. Netw. 2 (1) (2011) $74-83$.

[7] M. S. Kuran, H. B. Yilmaz, T. Tugcu, A tunnel-based approach for signal shaping in molecular communication, in: Proc. IEEE Int. Conf. on Commun. (ICC), 2013, pp. 776-781.

[8] T. Nakano, T. Suda, M. Moore, R. Egashira, A. Enomoto, K. Arima, Molecular communication for nanomachines using intercellular calcium signaling, in: Proc. IEEE Int. Conf. on Nanotechnol. (NANO), 2005, pp. 478-481.

[9] M. Kuran, T. Tugcu, B. Edis, Calcium signaling: Overview and research directions of a molecular communication paradigm, IEEE Wireless Commun. $19(5)$. 
[10] W. Guo, C. Mias, N. Farsad, J.-L. Wu, Molecular versus electromagnetic wave propagation loss in macro-scale environments, IEEE Trans. Molecular, Bio. and Multi-Scale Commun. 1 (1) (2015) 18-25.

[11] K. Srinivas, A. W. Eckford, R. S. Adve, Molecular communication in fluid media: The additive inverse gaussian noise channel, IEEE Trans. Inf. Theory 58 (7) (2012) 4678-4692.

[12] H. B. Yilmaz, A. C. Heren, T. Tugcu, C.-B. Chae, Three-dimensional channel characteristics for molecular communications with an absorbing receiver, IEEE Commun. Lett. 18 (6) (2014) 929-932.

[13] A. Akkaya, H. B. Yilmaz, C.-B. Chae, T. Tugcu, Effect of receptor density and size on signal reception in molecular communication via diffusion with an absorbing receiver, IEEE Commun. Lett. 19 (2) (2015) 155-158.

[14] M. Pierobon, I. F. Akyildiz, A physical end-to-end model for molecular communication in nanonetworks, IEEE J. Sel. Areas Commun. 28 (4).

[15] D. Kilinc, O. B. Akan, Receiver design for molecular communication, IEEE J. Sel. Areas Commun. 31 (12) (2013) 705-714.

[16] A. Noel, D. Makrakis, A. Hafid, Channel impulse responses in diffusive molecular communication with spherical transmitters, arXiv preprint arXiv:1604.04684.

[17] I. Llatser, D. Demiray, A. Cabellos-Aparicio, D. T. Altilar, E. Alarcon, N3sim: Simulation framework for diffusion-based molecular communication nanonetworks, Elsevier Simul. Model. Pract. Theory 42 (2014) 210-222.

[18] L. Felicetti, M. Femminella, G. Reali, A simulation tool for nanoscale biological networks, Elsevier Nano Commun. Netw. 3 (1) (2012) 2-18.

[19] H. B. Yilmaz, C.-B. Chae, Simulation study of molecular communication systems with an absorbing receiver: Modulation and isi mitigation techniques, Elsevier Simul. Model. Pract. Theory 49 (2014) 136 - 150.

[20] A. Noel, K. C. Cheung, R. Schober, D. Makrakis, A. Hafid, Simulating with accord: Actor-based communication via reaction-diffusion, Elsevier Nano Commun. Netw. 11 (2017) 44-75.

[21] B. Chopard, L. Frachebourg, M. Droz, Multiparticle lattice gas automata for reaction diffusion systems, Int. J. Modern Physics C 5 (01) (1994) 47-63.

[22] B. Chopard, A. Dupuis, A. Masselot, P. Luthi, Cellular automata and lattice boltzmann techniques: An approach to model and simulate complex systems, Adv. in Complex Sys. 5 (02n03) (2002) 103-246.

[23] J. Vidal Rodriguez, et al., Stochasticity in signal transduction pathways, Computational Science Lab, 2009. 
[24] K. Burrage, P. M. Burrage, A. Leier, T. Marquez-Lago, D. V. Nicolau, Stochastic simulation for spatial modelling of dynamic processes in a living cell, in: Design and Analysis of Biomolecular Circuits, Springer, 2011, pp. $43-62$.

[25] D. T. Gillespie, A general method for numerically simulating the stochastic time evolution of coupled chemical reactions, J. Comput. Physics 22 (4) (1976) 403-434.

[26] D. T. Gillespie, Exact stochastic simulation of coupled chemical reactions, J. Physical Chem. 81 (25) (1977) 2340-2361.

[27] E. Gul, B. Atakan, O. B. Akan, Nanons: A nanoscale network simulator framework for molecular communications, Elsevier Nano Commun. Netw. 1 (2) (2010) 138-156.

[28] H. B. Yilmaz, I. Demirkol, Voxel-based solver for diffusion-based molecular communications, in: Int. Workshop on Molecular Commun. (MOLCOM), 2018, pp. 1-2.

[29] M. S. Kuran, H. B. Yilmaz, T. Tugcu, I. F. Akyildiz, Modulation techniques for communication via diffusion in nanonetworks, in: Proc. IEEE Int. Conf. on Commun. (ICC), 2011, pp. 1-5. 